\title{
Nondeterministic decision tables in process control
}

\author{
V RAJARAMAN* and N R GARUD ${ }^{\dagger}$ \\ *Jawaharlal Nehru Centre for Advanced Scientific Research and Indian Institute \\ of Science, Bangalore 560 012, India \\ ${ }^{\dagger}$ Department of Electrical Engineering Indian Institute of Technology, Kanpur \\ 208016, India \\ email: rajaram@cadl.iisc.ernet.in
}

\begin{abstract}
In this paper we define nondeterministic decision tables to describe process control rules specified imprecisely. An example of such a control rule is "if temperature is high and pressure is low then open valve slightly". The definition of nondeterministic decision tables is based on fuzzy sets and associated logic. We show how nondeterministic decision tables are interpreted and specified actions executed based on measured values of independent control variables. When nondeterministic decision tables are formulated based on rules given by experts it is necessary to determine whether they have any redundant rules, missing rules or contradictory rules. We define these terms for nondeterministic decision tables and show how such logical errors can be detected in certain cases.
\end{abstract}

Keywords. Decision tables; fuzzy logic; logical errors; process control rules.

\section{Introduction}

Decision tables (Rajaraman 1987,1991 ) have been used extensively to specify complex logical procedures. The conditions specified in decision tables (DTs) are boolean, i.e. they can either take a 'yes' or a 'no' answer. Such DTs cannot handle situations where conditions are not precisely specifiable. A decision rule: "If temperature is high and pressure is low then open valve slightly", for instance, is intractable in boolean logic. Very often experienced operators of plants inuntiate decision rules in such loose nondeterministic terms. In this paper we define Nondeterministic Decision Tables (NDT) to reflect imprecisely defined decision rules. The definition is based on fuzzy sets and associated logic (Zadeh 1965; Kaufmann 1975). When a DT is formulated based on rules given by human experts it is necessary to determine whether the rules are complete, specify whether there are any redundant rules or any rules that are contradictory. These concepts are well defined for DTs based on boolean logic (Rajaraman 1987). In this paper these terms have been defined for an NDT. The ideas suggested here are of particular relevance in defining DTs for process control. 
Decision table formulation based on fuzzy sets have been proposed earlier by Francioni \& Kandel (1988). Fuzzy decision tables (FDT) as proposed by them are not suitable for specifying nondeterministic rules as in their formulation

- the input space is segmented into blocks via fuzzy variables defined on it. Inputs falling in a block cannot be differentiated and actions change abruptly across boundaries of blocks. Human judgement in general and process control in particular do not undergo abrupt changes - one finds a gradual phasing out of one action into another.

- Very often one finds the following phenomenon in a human-controlled process:

1. The action itself is fuzzy, i.e. the 'strength' of an action depends on the 'strength' with which a particular input satisfies a particular decision rule.

2. The action is the weighted mean of actions entailed by control rules satisfied by the inputs.

These aspects of process control cannot be supported by the FDT model suggested earlier (Francioni \& Kandel 1988). An added advantage of the model suggested in this paper is that it can be reduced to FDT (Francioni \& Kandel 1988) merely by putting a threshold by a process described later. Further the concept of incompleteness, redundancy and contradiction in fuzzy decision tables have not been analyzed earlier in the literature.

This paper is organized as follows. In the next section we describe how nondeterministic decision tables are defined and interpreted. In $\$ 3$ we define incomplete, redundant and contradictory specifications in relation to NDTs and how such errors are detected. The last section states the conclusions of the paper.

\section{Nondeterministic decision tables}

A DT, be it crisp or fuzzy, defines a logical procedure by means of a set of conditions and their related actions. A DT is divided into four quadrants. All conditions relevant to the procedure are listed in the condition stub (sometimes condition listing carries over to the 'condition entry' column). Such DTs are called extended entry DT (EEDT). NDTs are almost always EEDTs. All actions relevant to the procedure are listed in the action stub. The next step is to determine which conditions taken together should lead to which action(s) and to record them on the right hand side as a series of decision rules.

Example 1. Consider the DT of figure 1. The control process here monitors the opening of a valve contingent on certain measurements.

An interesting observation is that this NDT has one boolean condition as well. But this should not be surprising as fuzzy logic being an extension of boolean logic should work in that restricted domain as well.

Let us take one judgemental rule from the table and consider the problem of giving a proper interpretation to the fuzzy terms involved and to interpret the rules using a suitable logic.

If temperature is $h \mathrm{ig} h$ and pressure is low and valve ' $\mathrm{B}$ ' is closed, then open valve 'A' slightly. 
Condition Stub

Condition Entries

\begin{tabular}{|l||c|c|c|c|c|c|}
\hline Temperature & Normal & High & High & Low & Low & E \\
\cline { 1 - 5 } Pressure & Normal & High & Low & High & Low & L \\
Valve 'B' closed & Y & - & Y & Y & Y & E \\
\hline
\end{tabular}

Action Stub

Action Entries

\begin{tabular}{|l||l|l|l|l|l|l|}
\hline Valve 'A' & $\begin{array}{l}\text { Do not } \\
\text { open }\end{array}$ & $\begin{array}{l}\text { Open } \\
\text { fully }\end{array}$ & $\begin{array}{l}\text { Open } \\
\text { slightly }\end{array}$ & $\begin{array}{l}\text { Open } \\
\text { Halfway }\end{array}$ & $\begin{array}{l}\text { Do not } \\
\text { Open }\end{array}$ & $\begin{array}{l}\text { Do } \\
\text { not } \\
\text { open }\end{array}$ \\
\hline
\end{tabular}

Figure 1. A decision table for controlling a valve.

The fuzzy linguistic qualifiers involved here are high, low and slightly. Before we can attempt to give an interpretation to these qualitative terms we must decide the universe for each term, i.e. the range of the variable that these linguistic terms qualify. In this case the variables are temperature, pressure and extent of valve opening. Let us, for illustration, choose arbitrary ranges for these variables.

Range of Temperature $T$ is $[150,1000]^{\circ} \mathrm{C}$.

Range of Pressure $P$ is $[1,10]$ atm.

Extent of Valve $A$ opening is in range [0,1]

Extent of Valve B opening is [0,1]. (For simplicity valve B assumed to have boolean states.)

On these universes the linguistic terms used can be defined using fuzzy sets (Zadeh 1965). These fuzzy definitions are flexible and user defined e.g.

( $T$ is high) is a fuzzy set $T h$ whose membership function is

$$
m_{T h}(T)=1+\frac{2}{\pi} \arctan \left(\frac{T-1000}{b}\right),
$$

where $\mathrm{b}=212.5$. This choice of $b$ gives $m_{T h}(787.5)=0.5$

The arctan function has a property that it rises very fast from 0 to 0.5 but the rise from 0.5 to 1 is much slower. This fact is exploited to model the fact that humans are able to differentiate the degree of 'hotness' better towards the hottest end of a temperature range as compared to the temperate zone. Correspondingly the membership grade of $T$ in $T h$ changes rapidly towards the higher end and is rather slowly varying in the temperate zone.

( $P$ is low) is a fuzzy set $P l$ given by:

$$
m_{P l}(P)=1-\frac{2}{\pi} \arctan \left(\frac{P-1}{d}\right) d=1.5
$$

This choice of $d$ gives $m_{P l}(2.5)=0.5$.

(Open valve slightly) is a fuzzy set $0 s$ given by

$$
m_{0 s}(y)=\exp \left[-0.5\left(\frac{y-0.25}{g}\right)^{2}\right] \quad g=0.14
$$


An exponential membership function is chosen for the fuzzy set open valve "slightly" to ensure that it is symmetric about $y=0.25$. The parameter $g$ provides a good control over the width of the set.

Using these definitions of the linguistic qualifiers one can cast the nondeterministic decision rule into the following fuzzy logic function (Zadeh 1965; Kaufmann 1975).

$$
(T \in T h) A N D(P \in P l) A N D(\text { Valve } B \text { closed }) \Rightarrow(y \text { in } O s)
$$

where $A N D$ is the fuzzy conjunction operator and $\Rightarrow$ is the fuzzy implication operator.

Various definitions of the same are possible. The definition used by us is

$$
\begin{aligned}
& (T \in T h) A N D(P \in P l)=\min \left[m_{T h}(T), m_{P l}(P)\right], \\
& (P \in P l) \Rightarrow(y \in O s)=\min \left[m_{P l}(P), m_{O s}(y)\right] .
\end{aligned}
$$

Thus this rule gives an output fuzzy set $C$ given by the membership function

$$
m_{c}(y)=\min \left[m_{O S}(y), m_{T h}(T), m_{P l}(P)\right] .
$$

If the action corresponding to this rule had been a crisp singleton set then the output set $C$ is a fuzzy set containing a single element with membership grade or 'confidence value' given by (1).

With this analysis one can address the problem of giving a meaningful interpretation to the NDT as a whole. For that we need some more definitions.

$$
\begin{aligned}
& T l: m_{T l}(T)=1-(2 / \pi) \arctan (T-150 / 150) \\
& T(\text { normal }): T_{n}: m_{T n}(T)=\exp \left[-0.5(T-575 / 362.5)^{2}\right] \\
& P(\text { high }): P h: m_{P h}(P)=1+(2 / \pi) \arctan (P-10 / 2.25) \\
& P(\text { normal }): P_{n}: m_{P n}(P)=\exp \left[-0.5(P-5.5 / 0.75)^{2}\right]
\end{aligned}
$$

The algorithm for analysing the NDT is as follows:

1. Generate a matrix $H$ of dimension $p \times n$ where

$p=$ number of entries in the condition stub

$n=$ number of decision rules

$h_{i j}=1$ if the $(i, j)$ entry of the DT is a 'don't care' entry

and $h_{i j}=0$ if the $(i, j)$ entry is not a 'don't care'

For figure 1 the $H$ matrix is given as:

$$
H=\left[\begin{array}{lllll}
0 & 0 & 0 & 0 & 0 \\
0 & 0 & 0 & 0 & 0 \\
0 & 1 & 0 & 0 & 0
\end{array}\right]
$$

2. For a given input data (in our case temperature, pressure and condition of valve B) evaluate the decision rules and fill the $\mathrm{H}$ matrix as follows:

$h i j=m_{A i j}(x)$ where $A i j$ is the fuzzy set corresponding to the $i$ th condition in the $j$ th decision rule and $x$ is the input corresponding to $A i j$ 
for example,

$$
\begin{aligned}
h_{11} & =m_{A 11}(x) \\
& =m_{T n}(T) .
\end{aligned}
$$

Therefore,

$$
A_{11}=T n \text { and } x=T \text {. }
$$

Let $\mathrm{T}=200^{\circ} \mathrm{C} ; \mathrm{P}=4 \mathrm{~atm}$ and Valve $\mathrm{B}=$ closed; then the $H$ matrix is given as

$$
H=\left[\begin{array}{lllll}
0.58 & 0.17 & 0.17 & 0.80 & 0.80 \\
0.13 & 0.23 & 0.30 & 0.23 & 0.30 \\
1.0 & 1.0 & 1.0 & 1.0 & 1.0
\end{array}\right]
$$

3. AND the entries in each of the columns. This conjunction is the fuzzy conjunction relation. This column operation leads to a $1 \times n$ matrix which is the output fuzzy set $\mathrm{C}^{\prime}$. If the actions are themselves fuzzy then $\mathrm{C}^{\prime}$ is a fuzzy set of fuzzy subsets.

$$
C^{\prime}=\left[\begin{array}{lllll}
0.13 & 0.17 & 0.17 & 0.23 & 0.30
\end{array}\right] .
$$

After evaluating $\mathrm{C}^{\prime}$ the action that has the maximum membership grade in $\mathrm{C}^{\prime}$ is performed. If we assign discrete values to the valve opening in $[0,1]$ then that action will be performed. We define "do not open" as 0 , open slightly as 0.25 , open halfway as 0.5 and open fully as 1.0 . In the current problem rule 5 is satisfied as its membership function is maximum and the valve is not opened.

Consider the following situation. The input condition is:

$T=450^{\circ} \mathrm{C}, \quad P=9.5 \mathrm{~atm}, \quad$ Valve B closed.

The $H$ matrix in this case is

$$
\begin{aligned}
H & =\left[\begin{array}{lllll}
0.94 & 0.23 & 0.23 & 0.29 & 0.29 \\
0.0 & 0.86 & 0.11 & 0.86 & 0.11 \\
1 & 1 & 1 & 1 & 1
\end{array}\right], \\
C^{\prime} & =\left[\begin{array}{lllll}
0.0 & 0.23 & 0.11 & 0.29 & 0.11
\end{array}\right] .
\end{aligned}
$$

$C_{4}^{\prime}$ is maximum. Thus rule 4 is satisfied and the controller opens valve halfway. Had this been a boolean DT it would have adjudged $T$ as normal

$$
\left(m_{T n}(T)=0.94>0.5 ; m_{T h}(T), m_{T l}(T)<0.5\right),
$$

and $P$ as high and invoked the 'else' rule and done nothing. The fuzzy controller on the other hand takes a weighted judgement taking all parameters in parallel. Although the temperature is lower than normal it decides that the exceptionally high value of pressure demands immediate action. This brings out a strong case for judgemental control of processes.

We will now examine a slightly more complicated example.

Example 2. The NDT figure 2 controls a system of valves that maintain the level of a reservoir. The inlet valve is uncontrollable. The outlet valves are $\mathrm{A}, \mathrm{B}, \mathrm{C}$ which have three possible states. Normal (0.5), Increased (1.0), Decreased (0.0). It is also desirable that flow be maintained as high as possible in the priority $\mathrm{A}>\mathrm{B}>\mathrm{C}$. 


\begin{tabular}{|c|c|c|c|c|c|c|c|c|}
\hline & & $R_{1}$ & $R_{2}$ & $R_{3}$ & $R_{4}$ & $R_{5}$ & $\overrightarrow{R_{6}}$ & $R_{7}$ \\
\hline$\overline{C_{1}}:$ & $\begin{array}{l}\text { (Flow in interval- } \\
\text { min.specified flow) }\end{array}$ & Pos. & Neg. & Neg. & - & - & - & - \\
\hline$C_{2}$ & Flow in interval $>0$ & - & $\mathrm{Y}$ & $\mathrm{Y}$ & - & - & $\mathrm{N}$ & $\mathbf{N}$ \\
\hline$C_{3}$ & $\begin{array}{l}\text { (Flow in last } 3 \text { int. } \\
- \text { min.spec.flow) }\end{array}$ & - & - & - & Neg. & Neg. & Neg. & Neg. \\
\hline$C_{4}:$ & $\begin{array}{l}\text { (Actual height } \\
\text { - Specified height) }\end{array}$ & - & Pos. & Neg. & Pos. & Neg. & Pos. & Neg. \\
\hline$\overline{A_{1}:}$ & Open valve $A$ & Inc. & Nor. & Nor. & Nor. & Dec. & $\overline{\text { Dec. }}$ & Dec. \\
\hline$A_{2}$ : & Open valve $\mathrm{B}$ & Inc. & Dec. & Nor. & Dec. & Dec. & Dec. & Dec \\
\hline$A_{3}:$ & Open valve $\mathrm{C}$ & Nor. & Dec. & Dec. & Dec. & Dec. & Dec. & Dec. \\
\hline$A_{4}:$ & Alarm 1 & $\mathrm{~N}$ & $Y$ & $\mathrm{~N}$ & $\mathrm{Y}$ & $\mathrm{Y}$ & $\mathrm{Y}$ & $\mathrm{N}$ \\
\hline$A_{5}:$ & Alarm 2 & N & $\mathrm{N}$ & $\mathbf{N}$ & $\mathbf{N}$ & $\mathrm{N}$ & $\mathrm{N}$ & $Y$ \\
\hline & Action sets & S1 & $\mathrm{S} 2$ & S3 & S4 & S5 & S6 & S7 \\
\hline
\end{tabular}

Inc.: Increase, Nor.: Normal, Dec.: Decrease

Figure 2. Reservoir level control.

The linguistic terms positive (pos.) and negative (neg.) are given by fuzzy sets defined on the real axis.

$$
\begin{aligned}
& m_{\text {pos }}(x)=\left[\begin{array}{lll}
0 & & x \leq-1 \\
0.5+ & (2 / \pi) \arctan (x) & |x| \leq 1 \\
1 & x \geq 1
\end{array}\right], \\
& m_{n e g}(x)=1-m_{p o s}(x) \text {. }
\end{aligned}
$$

The universes of the various variables involved can be suitably normalized to conform to this universal definition. This method becomes simpler if the same linguistic terms qualify all variables as against defining separate sets for all variables.

Let us prescribe some arbitrary specification.

Minimum specified flow $f=$

Normalized flow in the interval $\left(: c_{1}\right)$

Cumulative flow over last three intervals

Normalized cumulative flow over last 3 intervals $\left(: c_{3}\right)$

Specified height of reservoir $=$

Normalized height $\left(: c_{4}\right)$
30

$\hat{f}=(f-30) / 20$

$f_{\text {cum }}$

$\hat{f}_{\text {cum }}=\left(f_{\text {cum }}-90\right) / 40$

5

$\hat{y}=(y-5) / 2$

At a particular sampling instant let the input measured values be:

$$
\begin{array}{lll}
f=27 & f_{\text {cum }}=77 & y=6.5 \\
\hat{f}=-0.15 & \hat{f}_{\text {cum }}=-0.32 & \hat{y}=0.75
\end{array}
$$


Using the values and the definitions of pos. and neg. given above, the $H$ matrix for this NDT becomes.

$$
\begin{aligned}
H & =\left[\begin{array}{lllllll}
0.4 & 0.6 & 0.6 & 1 & 1 & 1 & 1 \\
1 & 1 & 1 & 1 & 1 & 0 & 0 \\
1 & 1 & 1 & 0.7 & 0.7 & 0.7 & 0.7 \\
1 & 0.91 & 0.09 & 0.91 & 0.09 & 0.91 & 0.09
\end{array}\right], \\
C^{\prime} & =\left[\begin{array}{lllllll}
0.4 & 0.6 & 0.09 & 0.7 & 0.09 & 0.0 & 0.0
\end{array}\right]
\end{aligned}
$$

The corresponding fuzzy action can be found out in two ways:

1. The output corresponding to the rule with the highest membership grade in $C^{\prime}$ is executed. In which case the action is corresponding to $C_{4}^{\prime}$.

Valve A : normal $\quad 0.5$

Valve B : decreased 0.0

Valve $C$ : decreased 0.0

Alarm 1: activated

2. The actions $A_{1}, \ldots, A_{5}$ can be considered independent of each other and the weighted sum of $c_{i}^{\prime}$ to be the action. Alarms, however, have to be activated with a threshold acting on their membership grade. The threshold chosen here is 0.5 . In this case the controller action is

Valve A: $\quad 0.58 \quad\left(\frac{1 \times .4+0.5 \times 0.6+0.5 \times .09+0.5 \times .7+0+0+0}{0.4+0.6+0.09+0.7+0.09}\right)=0.58$

Valve B: $\quad 0.24$

Valve C: $\quad 0.10$

Alarm 1: $\quad$ activated

Alarm 2: $\quad$ not activated

Consider the same process controlled by a boolean DT. In this DT the sets positive and negative shall be defined as

$$
\begin{aligned}
m_{\text {pos }}(x) & =1 \text { if } x>0 \\
& =0 \text { if } x \leq 0, \\
m_{\text {neg }}(x) & =1-m_{\text {pos }}(x) .
\end{aligned}
$$

R2 and R4 would have both been satisfied. Fortunately the actions entailed by both are same. In fact NDT with method 1 also suggests the same action.

Method 2, however, is better than the boolean DT by keeping both B and C open, albeit at low levels. Thus NDT is able to maintain the conflicting demands of level maintenance and flow maintenance in a better manner.

There are many pathological cases which hamper the efficient working of an NDT. One such case is where the fuzzy table does not categorize a particular input condition to any of the actions strongly.

The other possibility is that a point gets strongly classified into two altogether different actions. What is to happen in this contradictory situation is the point of discussion of the next section. 


\section{Concept of incompleteness, redundancy and contradictory specification in NDTs}

These concepts have been borrowed from deterministic DTs where they have been shown to be worthwhile in properly designing algorithms to implement a set of rules (Rajaraman 1987). Let us understand what each of these terms imply:

Incompleteness: This refers to a situation wherein there is no entry for a logically allowable combination of conditions.

Redundancy: Let $R_{1}$ : if $A_{1}$ then $B_{1}$, $R_{2}$ : if $A_{2}$ then $B_{1}$,

but from the conditions in the problem there is a constraint if $A_{2}$ then $A_{1}$. In such situations rule $R_{2}$ is redundant.

Contradictory specifications: This situation occurs when the DT demands 'disjoint' actions (i.e. actions distinct and incompatible) for a particular input data.

Before trying to implement these concepts on a NDT let us understand the restrictions that bind us.

1. The rule set should be clearly defined, i.e. there should not be any 'else' rule.

2. The actions should be crisp and discrete

3. There should be a threshold defined on the membership grade that a particular action has in the output fuzzy set. Without this restriction the DTs are by their nature complete.

The idea of a 'threshold' can be formalized via the $\alpha$ cut operation of fuzzy sets (Kaufmann 1975). The $\alpha$-cut of fuzzy set $A$ (designated by $A_{\alpha}$ ) is defined as

$$
A_{\alpha}=\left\{x \in U \mid m_{A}(x) \geq \alpha\right\} \text {. }
$$

$A_{\alpha}$ is therefore a crisp set $\subseteq \mathrm{U}$. By putting a threshold of $\alpha$ on $A$ we get $A_{\alpha}$. The $\alpha$-cut operation preserves the set operations ie.

$$
\begin{aligned}
(A \cap B)_{\alpha} & =A_{\alpha} \cap B_{\alpha}, \\
(\vec{A})_{\alpha} & =\vec{A}_{\alpha}, \\
(A \cup B)_{\alpha} & =A_{\alpha} \cup B_{\alpha},
\end{aligned}
$$

with $\cap, \cup$ and ${ }^{-}$suitably defined for crisp and fuzzy sets (Kaufmann 1975). Thus $\alpha$-cut operation can be looked upon as a homomorphism from the set of fuzzy subsets to ordinary sets. This property has an important bearing on our problem.

Let $\mathrm{R}$ : If $x \in A$ and $y \in B$ and $z \in C$ then let $w=A_{n}$ be a fuzzy decision rule. $\mathrm{A}, \mathrm{B}, \mathrm{C}$ are fuzzy sets; $A_{n}$ is a crisp action.

Now if we impose a threshold on the output fuzzy set of the NDT to which $R$ belongs then

$$
\begin{aligned}
m_{c^{\prime}}\left(A_{n}\right)= & 1 \Longleftrightarrow \min \left[m_{A}(x), m_{B}(y), m_{c}(z)\right] \geq \alpha \\
& \text { where } \alpha \text { is the threshold } \\
& \Longleftrightarrow\left[\left(m_{A}(x) \geq \alpha\right) \wedge\left(m_{B}(x) \geq \alpha\right)\right. \\
& \left.\wedge\left(m_{c}(x) \geq \alpha\right)=1\right] \\
& \Longleftrightarrow\left[\left(x \in A_{\alpha}\right) \wedge\left(y \in B_{\alpha}\right) \wedge\left(z \in C_{\alpha}\right)=1\right] .
\end{aligned}
$$


i.e., putting threshold on the output set can be equivalently viewed as putting thresholds on the fuzzy sets underlying the condition. Once thresholds are put on fuzzy sets they get mapped to their homomorphic crisp sets. The long established techniques of validation of such DTs (Rajaraman 1987) can now be used.

Since the homomorphic images of fuzzy sets are going to depend on the threshold chosen, it is quite natural to believe that the conclusions will depend on the level of threshold: $\alpha$ chosen. The steps for analyzing an NDT are:

1. Set up the threshold and $\alpha$-cuts of the fuzzy sets underlying the conditions.

2. Draw the Karnaugh map of the boolean DT so arrived at as explained by Rajaraman (1987)

- Cross out all squares in the Karnaugh map that are logically impossible

- Fill out the actions dictated by the table

- All entries of an EEDT are considered separate conditions

- Look for real or apparent contradictions

We shall consider example 2 again to illustrate these steps. The threshold is set at 0.5 in which case

$$
\begin{aligned}
\text {Pos}_{\alpha} & =\{x \text { real } \mid x \geq 0\} \text { and } \\
\text { Neg }_{\alpha} & =\{x \text { real } \mid x<0\}
\end{aligned}
$$

Let us designate action sets corresponding to $R_{1}, R_{2}, \ldots, R_{7}$ as $S_{1}, S_{2}, \ldots, S_{7}$. There is one logically impossible condition i.e. $C_{1}=Y$ and $C_{2}=N$ as the minimum specified flow in the interval is positive. The Karnaugh map of the DT is given in figure 3.

The crossed out area is logically impossible under the constraints of the problem. The two empty boxes imply there are input combinations for which no rule is satisfied with a membership grade $\alpha$, i.e. the table is incomplete when the threshold is set at 0.5 .

Boxes containing two or more actions are potential sites of contradition. Contraction may be apparent as in the case of $S_{2}$ and $S_{4}$ as both actions are the same. Other contradictions are all real contradictions.

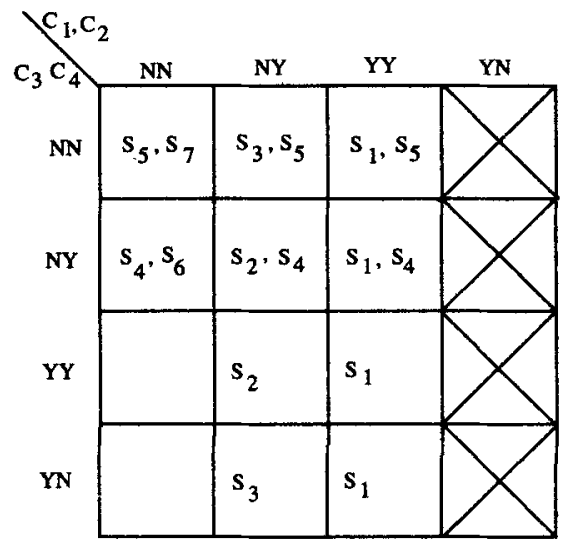

Figure 3. Karnaugh map for the NDT of figure 2. 


\begin{tabular}{l||ccccc}
\hline & $R_{1}$ & $R_{2}$ & $R_{3}$ & $R_{4}$ & $R_{5}$ \\
\hline$C_{1}:$ Temperature & High & High & Low & - & - \\
$C_{2}:$ V1 open & $\mathrm{Y}$ & $\mathrm{Y}$ & $\mathrm{Y}$ & - & $\mathrm{Y}$ \\
$C_{3}:$ V2 open & - & - & $\mathrm{N}$ & $\mathrm{Y}$ & - \\
$C_{4}:$ Pressure & - & High & - & High & Low \\
\hline \hline Action: Open V & Full & Full & Slightly & Halfway & Slightly \\
& $\mathrm{A} 1$ & $\mathrm{~A} 2$ & $\mathrm{~A} 3$ & $\mathrm{~A} 4$ & $\mathrm{~A} 5$ \\
\hline
\end{tabular}

Figure 4. NDT for example 3.

No redundancy can be seen. If there is any redundancy then the area occupied by actions corresponding to any one rule shall be completely covered by another. Since this is not so there is no redundancy.

Example 3. Consider another simple example. The NDT in figure 4 controls opening of valve $V$ depending on temperature pressure and states of two other valves $V_{1}$ and $V_{2}$.

Definition of the linguistic qualifiers as fuzzy sets is:

Temperature (T): $0 \leq T<1000^{\circ} \mathrm{C}$

$$
\begin{aligned}
T h: m_{T h}(T) & = \begin{cases}0 & T<200 \\
0.5+\frac{2}{\pi} \arctan \left(\frac{T-500}{300}\right) & T \epsilon[200,800] \\
1 & T>800\end{cases} \\
T l: m_{T l}(T) & =1-m_{T h}(T)
\end{aligned}
$$

Pressure(P): $O<P<500 \mathrm{~kg} \mathrm{~cm}^{-2}$

$$
\begin{aligned}
P h: m_{P h}(P) & = \begin{cases}0 & P<150 \\
0.5+\arctan \left(\frac{P-250}{100}\right) & P \epsilon[150,350] \\
1 & P>350\end{cases} \\
P l: m_{P l}(P) & =1-m_{P h}(P)
\end{aligned}
$$

Consider rules $R_{1}$ and $R_{2}$ : The actions $A_{1}$ and $A_{2}$ are the same written out in terms of fuzzy logic operators. These rules are:

$$
\begin{aligned}
& R_{1}:(T \epsilon T h) \text { and }(V 1 \text { open }) \Rightarrow \text { open } V \text { full } \\
& R_{2}:(T \in T h) \text { and }(V 1 \text { open }) \text { and }(P \in P h) \Rightarrow \text { open } V \text { full. }
\end{aligned}
$$

Consider membership grades of the outputs corresponding to these rules in $C^{\prime}$

$$
\begin{aligned}
m_{01}(y) & =\min \left(m_{T h}(T), m_{\text {open }}(V 1)\right) \\
m_{02}(y) & =\min \left(m_{T h}(T), m_{\text {open }}(V 1), m_{P h}(P)\right) \\
& \leq \min \left(m_{T h}(T), m_{\text {open }}(V 1)\right)=m_{01}(y)
\end{aligned}
$$

This implies that rule $R_{1}$ will completely cover $R_{2}$ whatever be the threshold. Thus $R_{2}$ is redundant. In the shortened table we shall drop out $R_{2}$. Take a threshold of 0.5 and draw the Karnaugh map of the shortened DT as shown in figure 5. 


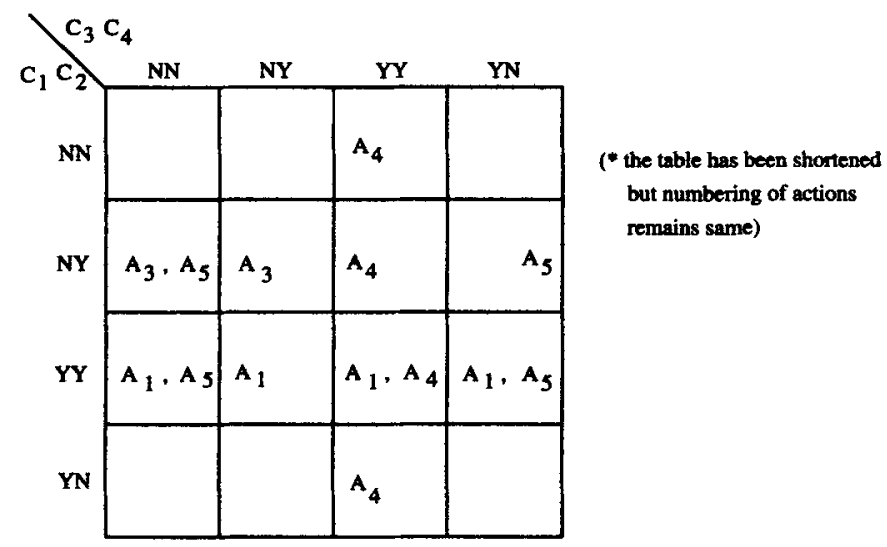

Figure 5. Karnaugh map for example 3.

The table as one can see is

- incomplete: as there are empty (uncrossed out) boxes.

- contradictory: as ( $A_{1}$ and $\left.A_{5}\right)$ and $\left(A_{1}\right.$ and $\left.A_{4}\right)$ are incompatible. ( $A_{3}$ and $\left.A_{5}\right)$ is not a real contradiction as they are both same.

- not redundant: as no reduction in rules is possible because there is no overlapping.

An interesting feature of this example is that redundancy was detected and removed without reference to a threshold. This procedure, although the most satisfying, is very difficult to execute.

So far in our discussion of fuzzy process control and implementation of NDT to control processes 'threshold' was never mentioned whereas this section is formulated with the 'threshold' as the crux of the whole matter. The connection between these apparently dichotomous ideas lies in the nature of human decsion making. Human decision rules, although not incomplete, are contradictory and often redundant. To be able to remove them one would have to impose some determinism via the threshold. By no means is this undesirable. It safeguards against some serious lacunae of the unconstrained NDTs.

1. Suppose for a particular input the highest membership grade is 0.2 . If there is no threshold a certain action would be taken. If threshold is put at 0.4 the controller Karnaugh map will indicate that it is incomplete and will caution the designer about it. It shall, therefore, prevent an action from being executed if its 'confidence value' is very low.

2. Two actions have a very close membership grades in the output set $C^{\prime}$. say 0.6 and 0.62 . The NDT will choose the action corresponding to 0.62 and execute it. A suitably chosen threshold will bring out this fact and warn the designer about the phenomenon mentioned above. This implies that two rules are closely allied and a restructuring of these rules might lead to a reduction in the number of rules.

Thus we see that choosing the right threshold for analysis is of utmost importance in proper design of NDTs. It is very subjective and changes from one application to another. A very low threshold means that the controller may be taking actions at very low confidence values. A very high threshold on the other hand forces the controller to take an action only 
if a rule is satisfied to a very high degree thereby denying the nonspecificity of conditions and tending towards a boolean DT. A judicious threshold has therefore, to be, chosen.

One thing must, however, be kept in mind. We have moved to a fuzzy controller because a boolean DT cannot work. The very fact implies that we can tolerate some amount of contradiction. If we want to exploit the judgemental nature of NDTs, thresholds must be used for design and never during implementation. It will be grossly erroneous to assume that an NDT is blind to all inputs that belong to a box showing a contradiction when threshold is set at $\alpha$. It is just that it is blind with $\alpha$ as the benchmark. Since action corresponding to the maximum membership grade is performed most of the contradiction will be resolved.

\section{Conclusions}

We have seen in this paper that nondeterministic decision tables (NDTs) capture the smooth control exercised by a human operator while transitioning between actions. It can cope well in situations where the aims may be contradictory and the control action requires a weighted judgement. In its attempt to capture the judgemental nature of human decision making it becomes vulnerable to drawbacks that go with fuzzy modelling of conditions. The concepts of incompleteness, redundancy and contradiction, that are so well defined for boolean DT and help in their design and validation, are not so well specified in case of NDTs. To be able to define them for NDTs one has to introduce the concept of threshold. The threshold, if applied to an NDT, kills the very spirit of fuzziness as it converts it into a boolean DT. However, a well chosen threshold (for choosing a threshold is highly subjective) and the analysis of the boolean DT can give the designer of the NDT an idea about its quality but cannot directly help him improve it.

The formulation of NDT presented here is much more general than the FDT suggested by Francioni \& Kandel (1988). The NDT given here reduces to FDT if thresholds are applied on the fuzzy sets to segment the input space into distinct blocks and the rules recalculated using the procedure given here. As stated earlier such a table is incapable of taking 'judgemental' decision crucial for process control.

Summing up, NDTs are good operational tools for process control but for them to be put on the same pedestal as DTs one would have to look for more satisfying concepts for defining their completeness etc., which not only evaluate but also suggest method for improving them.

Grant of a fellowship to N R Garud by the Jawaharlal Nehru Centre for Advanced Scientific Research, Bangalore for doing this research is gratefully acknowledged. The authors thank an anonymous referee for constructive comments.

\section{References}

Francioni J M, Kandel A 1988 A software engineering tool for expert system design. IEEE Expert 3: $33-41$

Kaufmann A 1975 Introduction to theory of fuzzy sets (New York: Academic Press) 
Rajaraman V 1987 Validation of decision tables used in process control. IEEE Trans. Ind. Electron. 34: $168-171$

Rajaraman V 1991 Decision tables. Encyclopedia of computer science and engineering (New York: Marcel Dekker) vol. 24, suppl. 9, pp 85-106

Zadeh L A 1965 Fuzzy sets. Inf. Contr. 8: 338-353 